\title{
Off-label Use of Itolizumab in Patients with COVID-19 ARDS: Our Clinical Experience in a Dedicated COVID Center
}

\author{
Poonam Kumari ${ }^{1} \oplus$, Amarjeet Kumar ${ }^{2} \odot$, Chandni $\operatorname{Sinh}^{3} \odot$, Ajeet Kumar ${ }^{4} \odot$, Prabhat K $\operatorname{Singh}^{5} \oplus$, SK Arun $^{6} \odot$
}

\begin{abstract}
Severe acute respiratory syndrome coronavirus 2 has affected millions of people worldwide. This pandemic requires newer medical management strategies to control the morbidity and mortality associated with the disease. Several approaches, including global targeting of inflammation or neutralizing a single key inflammatory mediator, are being employed to cope with cytokine storms in coronavirus disease-2019 (COVID-19). The role of anti-inflammatory biologics, such as acalabrutinib, tocilizumab, anakinra, and itolizumab can become relevant. Itolizumab is a humanized recombinant immunoglobulin G1 monoclonal antibody. It targets the extracellular, scavenger receptor cysteine-rich (SRCR) distal domain 1 of CD6 and is responsible for priming, activation, and differentiation of T-cells. Itolizumab has been approved by the Drug Controller General of India for the treatment of COVID-19 in India. Here, we shared our clinical experience of 20 patients having moderate acute respiratory distress syndrome (ARDS) due to COVID-19 on treatment with itolizumab. We observed the mortality benefit with single-dose itolizumab (1.6 mg/kg) in patients having moderate COVID-19 ARDS.
\end{abstract}

Keywords: Acute respiratory distress syndrome (ARDS), COVID-19, Cytokine storm, Itolizumab, Monoclonal antibody. Indian Journal of Critical Care Medicine (2021): 10.5005/jp-journals-10071-23787

\section{HIGHLIGHTS}

- Fifteen percent of coronavirus disease-2019 (COVID-19) patients who develop acute respiratory distress syndrome within a week after the appearance of symptoms.

- Cytokine storm is a life-threatening acute systemic inflammatory syndrome, which is characterized by multiorgan failure and fever.

- Itolizumab is an anti-CD6 receptor monoclonal antibody which seems to be the possible strategy for patients with cytokine storm.

- We used Itolizumab off-label in patients with severe COVID19 from August to September 2020 based on its hypothetical benefit and report the experience in our institute AlIMS, Patna.

\section{INTRODUCTION}

Severe acute respiratory syndrome (SARS) coronavirus 2 (CoV-2) has affected millions of people worldwide. This pandemic requires newer medical management strategies to control the morbidity and mortality associated with the disease. About $15-20 \%$ of the affected patients develop pulmonary symptoms, such as breathing difficulty, and require hospital admission for oxygen therapy and supportive care. ${ }^{1}$ There is a marked increase in the level of cytokines (e.g., interleukin(IL)-1B, IL-6, and IL-12) and chemokines and this is referred to as "cytokine storm." ${ }^{2}$ There is an elevation of other markers of inflammation, coagulation, and organ damage, such as C-reactive protein (CRP), D-dimer, lactate dehydrogenase, ferritin, and troponin-I. Hence, anti-inflammatory agents, such as acalabrutinib, tocilizumab, anakinra, and itolizumab might have a role to play in this disease. ${ }^{3-8}$ Itolizumab is a humanized recombinant immunoglobulin $\mathrm{G} 1$ monoclonal antibody. It targets extracellular, scavenger receptor cysteine-rich distal domain 1 of
$1,3,4,6$ Department of Anaesthesiology, All India Institute of Medical Sciences, Patna, Bihar, India

${ }^{2}$ Department of Trauma and Emergency, All India Institute of Medical Sciences, Patna, Bihar, India

${ }^{5}$ Director, All India Institute of Medical Sciences, Patna, Bihar, India

Corresponding Author: Amarjeet Kumar, Department of Trauma and Emergency, All India Institute of Medical Sciences, Patna, Bihar, India, Phone: +91 09570890646, e-mail: amarjeetdmch@gmail.com

How to cite this article: Kumari P, Kumar A, Sinha C, Kumar A, Singh PK, Arun SK. Off-label Use of Itolizumab in Patients with COVID-19 ARDS: Our Clinical Experience in a Dedicated COVID Center. Indian J Crit Care Med 2021;25(4):467-469.

Source of support: Nil

Conflict of interest: None

CD6 and is responsible for priming, activation, and differentiation of T-cells. ${ }^{9}$ Drug Controller General of India (DCGI) has approved itolizumab for the treatment of COVID-19 in India. Here, we share our single-center clinical experience of off-label use of this drug in 20 patients with acute respiratory distress syndrome (ARDS) due to COVID-19.

\section{Materials and Methods}

This is our single-center clinical experience of patients infected with COVID-19 and treated with itolizumab between August and September 2020 at All India Institute of Medical Sciences, Patna. We recorded various clinical and biochemical parameters every day from the day of admission to 14 days after administration of itolizumab. Clinical parameters, such as respiratory rate and oxygen supplementation, were measured daily. Laboratory parameters 
[CRP, lymphocytes, aspartate aminotransferase (AST), and alanine aminotransferase (ALT)] were measured at the clinician's discretion and collected from baseline to day 14 .

Due to high costs, lack of knowledge, and safety risks, a multidisciplinary ethics committee validated the off-label use of itolizumab in moderately severe patients. Patient consent was requested and marked in the patients' records.

The patients who received itolizumab had the following criteria:

- Age between 18 years and 60 years

- Virological diagnosis of SARS-CoV2 infection (polymerase chain reaction)

- Patients who were suffering from moderate COVID-19 ARDS (as defined by $\mathrm{PaO}_{2} / \mathrm{FiO}_{2}$ ratio of $<200$, or more than $25 \%$ deterioration from the immediate previous value within 24 hours in patients having more than $\mathrm{PaO}_{2} / \mathrm{FiO}_{2}>200$ ).

- Serum ferritin level $\geq 400 \mathrm{ng} / \mathrm{mL}$ at baseline or IL-6 levels greater than four times the upper limit of normal.

Exclusion criteria for injection itolizumab included: patients with a history of severe allergic reactions to monoclonal antibodies, history of active or latent tuberculosis (TB) infection, patients on oral immunosuppressive drugs within the past 6 months, pregnant or breastfeeding patients, or those with a positive pregnancy test in a predose examination. Patients with a known history of hepatitis $B$, hepatitis $\mathrm{C}$, or HIV, absolute neutrophil count $<1000 / \mathrm{mm}^{3}$, platelets $<50000 / \mathrm{mm}^{3}$, absolute lymphocyte count $<500 / \mathrm{mm}^{3}$, very severe ARDS $\mathrm{PaO}_{2} / \mathrm{FiO}_{2} \leq 100$ with positive endexpiratory pressure (PEEP) $\geq 5$, and ARDS due to other secondary infections/bacterial sepsis (high procalcitonin value) were also not administered the drug.

Patients of itolizumab group received the drug at a dose of $1.6 \mathrm{mg} / \mathrm{kg}$. Itolizumab was dissolved in $250 \mathrm{~mL}$ of $0.9 \%$ normal saline. The infusion was started at a rate of $25 \mathrm{~mL}$ in the first hour and given over a time period of 4-6 hours. During infusion, vitals of the patient were monitored. Premedication with hydrocortisone $100 \mathrm{mg}$ and pheniramine $30 \mathrm{mg}$ intravenous was given 30 minutes before infusion. The second dose of the drug was repeated according to clinical evaluation, response to the first dose, and lab parameters. All patients admitted to the intensive care unit (ICU) received standard baseline treatments as per SOP AIIMS, Patna. Antibiotics were given as per antibiogram report. During hospitalization, these patients required oxygen therapy in the form of nonrebreathing mask (NRM), high-flow nasal cannula (HFNC), continuous positive airway pressure (CPAP), or invasive (i.e., mechanical ventilation).

In this clinical experience, we used itolizumab in a very small subset of patients admitted in COVID ICU. This allowed us to compare the effect of itolizumab versus control. We compared the parameters in both groups of these patients: one treated with itolizumab and the other with a relatively similar clinical impairment who had not been treated with any other immunomodulators.

We have limited crude raw data of patients who have not received itolizumab. Bias could not be eliminated in the groups due to presence of sicker patients with comorbidities in the control group.

\section{Results}

\section{Patients' Outcome}

From August 1, 2020, to September 30, 2020, totally 150 patients were included in our study. Of these 150, 20 received injection itolizumab $(1.6 \mathrm{mg} / \mathrm{kg})$ following the fulfillment of inclusion criteria. Among these patients who received itolizumab, seven patients were not survived and 1-month mortality rate was 35\%. Among these patients who have not received itolizumab were 130 and 1 -month mortality was $60 \%$.

\section{Adverse Effects}

We detected infusion-related adverse reactions in eight patients; six patients have mild shivering and other two patients noted tachycardia and oxygen desaturation, for which they required increment of oxygen support.

\section{Discussion}

There are various drugs like tocilizumab, itolizumab, and anakinra used in the treatment of COVID-19. Among these, itolizumab is a new biological agent that has been approved in India for the treatment of psoriasis. ${ }^{10}$ It acts by immunomodulating T-effector function and its migration to the inflammation site, sparing Tregs, and preserving the antiviral response. It acts upstream at the Th1 and Th17 pathways, ${ }^{9,11}$ i.e., at the T-effector cells. Thus, it decreases the release of multiple cytokines and cell signaling transduction factors primarily involving the Th17 and Th1 pathways. Other drugs, such as tocilizumab or anakinra, block cytokines at a downstream level. Itolizumab also binds to CD6 receptor and blocks activated leukocyte cell adhesion molecule-mediated T-cell activation. For this reason, it is used in the treatment of COVID-19 patient clinical trial done in Cuba. ${ }^{12}$ Tocilizumab was also used in the treatment of COVID-19 patients with moderate-to-severe ARDS. ${ }^{13}$ Albertini et al. ${ }^{14}$ in an observational study over off-label use of tocilizumab concluded that tocilizumab appears to be effective in decreasing oxygen withdrawal time and respiratory failure symptoms, avoiding mechanical ventilation, and stopping the biological inflammatory process of COVID-19 pneumonia in hospitalized patients, especially in those with $>50 \%$ of initial lung lesions on computed tomography scan. It is important to mention that IL- 6 is not the appropriate marker of disease improvement as the soluble IL- 6 receptor immune complex has a long half-life. It remains in the serum for a long time because of its long half-life after tocilizumab administration. IL- 6 levels might also increase after tocilizumab administration because of less consumption of IL-6. ${ }^{13}$

We observed mortality benefit (35 vs 60\%) in moderate COVID19 ARDS patients who have received single-dose itolizumab $1.6 \mathrm{mg} / \mathrm{kg}$ in comparison to patients who have not received immunomodulator therapy (tocilizumab/itolizumab) in the course of COVID-19 disease. In India, the mortality rate in moderate-tosevere COVID-19 pneumonia is $42 \% .^{15}$ Tocilizumab was another immunomodulator used at our center. In our experience, despite giving two doses within 12 hours we have found no improvement. We also observed cost benefit in patients receiving itolizumab, as a single dose was sufficient.

A randomized, controlled, open-label study was conducted by Biocon at four hospitals in India, in moderate-to-severe COVID19 ARDS patients. As reported by Biocon, the mortality benefit observed in the itolizumab arm was statistically significant than the only supportive care group.

\section{Limitations}

Here, we have shared our clinical experience with moderate COVID ARDS patients on treatment with itolizumab. We have not compared the clinical outcome of patients on treatment with other 
drugs like tocilizumab. It would be difficult to comment on or to speculate that itolizumab has better survival benefits without a well-conducted randomized controlled trial, comparing various modalities. Nonetheless, this clinical experience does make us consider itolizumab the treatment option. We have not included patients with mild and severe ARDS $\left(\mathrm{PaO}_{2} / \mathrm{FiO}_{2} \leq 100\right.$ with $\left.\mathrm{PEEP} \geq 5\right)$. Due to unequal distribution of comorbidities among patients, we acknowledge that our results should be interpreted with caution as significant heterogeneity exists within these factors.

\section{Conclusion}

We observed mortality benefit with single-dose itolizumab $(1.6 \mathrm{mg} / \mathrm{kg})$ in patients having moderate COVID-19 ARDS. A wellstructured comparative study is required to validate our result and to find superiority over tocilizumab. Use and recommendations for itolizumab in COVID-19 moderate/severe disease should be taken with caution as not only the supportive data are lacking but also long-term outcomes of itolizumab in COVID-19 diseases are yet to be known.

\section{OrCID}

Poonam Kumari @ https://orcid.org/0000-0003-0118-2187

Amarjeet Kumar (1) https://orcid.org/0000-0002-4272-5750

Chandni Sinha @ https://orcid.org/0000-0002-4107-2671

Ajeet Kumar 10 https://orcid.org/0000-0002-1464-6684

Prabhat K Singh (1) https://orcid.org/0000-0003-1888-4679

Arun SK 이 https://orcid.org/0000-0002-9788-3350

\section{References}

1. Wang D, Hu B, Hu C, Zhu F, Liu X, Zhang J, et al. Clinical characteristics of 138 hospitalized patients with 2019 novel coronavirus-infected pneumonia in Wuhan, China. JAMA 2020;323(11):1061-1069. DOI: 10.1001/jama.2020.1585.

2. Coperchini F, Chiovato L, Croce L, Magri F, Rotondi M. The cytokine storm in COVID-19: An overview of the involvement of the chemokine/chemokine-receptor system. Cytokine Growth Factor Rev 2020;53:25-32. DOI: 10.1016/j.cytogfr.2020.05.003.

3. Roschewski M, Lionakis MS, Sharman JP, Roswarski J, Goy A, Monticelli $M A$, et al. Inhibition of Bruton tyrosine kinase in patients with severe COVID-19. Sci Immunol 2020;5(48):eabd0110. DOI: 10.1126/ sciimmunol.abd0110.

4. Alijotas-Reig J, Esteve-Valverde E, Belizna C, Selva-O'Callaghan A, Pardos-Gea J, Quintana A, et al. Immunomodulatory therapy for the management of severe COVID-19. Beyond the anti-viral therapy: a comprehensive review. Autoimmun Rev 2020;19(7):102569. DOI: 10.1016/j.autrev.2020.102569.

5. Marovich M, Mascola JR, Cohen MS. Monoclonal antibodies for prevention and treatment of COVID-19. JAMA 2020;324(2):131-132. DOI: 10.1001/jama.2020.10245.

6. Bughani U, Saha A, Kuriakose A, Nair R, Sadashivarao RB, Venkataraman $R$, et al. T cell activation and differentiation is modulated by a CD6 domain 1 antibody Itolizumab. PLoS One 2017;12(7):e0180088. DOI: 10.1371/journal.pone.0180088.

7. Guaraldi G, Meschiari M, Cozzi-Lepri A, Milic J, Tonelli R, Menozzi M, et al. Tocilizumab in patients with severe COVID-19: a retrospective cohort study. Lancet Rheumatol 2020;2(8):e474-e484. DOI: 10.1016/ S2665-9913(20)30173-9.

8. Cavalli G, De Luca G, Campochiaro C, Della-Torre E, Ripa $M$, Canetti $D$, et al. Interleukin-1 blockade with high-dose anakinra in patients with COVID-19, acute respira- tory distress syndrome, and hyperinflammation: a retrospective cohort study. Lancet Rheumatol 2020;2(6):e325-e331. DOI: 10.1016/S26659913(20)30127-2.

9. Nair P, Melarkode R, Rajkumar D, Montero E. CD6 synergistic co-stimulation promoting proinflammatory response is modulated without interfering with the activated leucocyte cell adhesion molecule interaction. Clin Exp Immunol 2010;162(1):116-130. DOI: 10.1111/j.1365-2249.2010.04235.x.

10. Gupta A, Sharma YK, Deo K, Kothari P. Severe recalcitrant psoriasis treated with itolizumab, a novel anti-CD6 monoclonal antibody. Indian J Dermatol Venereol Leprol 2016;82(4):459-461. DOI: 10.4103/0378-6323.181466.

11. Anand A, Assudani D, Nair P, Krishnamurthy S, Deodhar S, Arumugam $M$, et al. Safety, efficacy and pharmacokinetics of T1h, a humanized anti-CD6 monoclonal antibody, in moderate to severe chronic plaque psoriasis - results from a randomized phase II trial. (96.13). J Immunol 2010 Apr 1;184(1 Supplement) 96.13 [Phase 2 trial of Itolizumab in moderate to severe chronic plaque psoriasis].

12. Treatment of patients with severe SARS-CoV-2 pneumonia with the anti-CD6 monoclonal antibody itolizumab-full text-WHO-ICTRP [Internet] [Cited $2020 \mathrm{Jul}$ 16]. Available from: https://apps.who.int/ trialsearch/Trial2.aspx?TrialID=RPCEC00000311.

13. Xu X, Han M, Li T, Sun W, Wang D, Fu B, et al. Effective treatment of severe COVID-19 patients with tocilizumab. Proc Natl Acad Sci U S A. 2020;117(20):10970-10975. DOI: 10.1073/pnas.2005615117.

14. Albertini L, Soletchnik M, Razurel A, Cohen J, Bidegain F, Fauvelle F, et al. Observational study on off-label use of tocilizumab in patients with severe COVID-19. Eur J Hosp Pharm 2020;0:1-7. DOI: 10.1136/ ejhpharm-2020-002414.

15. Fewer COVID-19 deaths in ICU suggest hospital care improving: study 2020 [Accessed: September 20, 2020]. Available from: https://www. ndtv.com/world-news/coronavirus-fewer-covid-19-deaths-in-icusuggest-hospital-care-improving-study-2264244. 